\title{
Shufengjiedu capsules protect against neuronal loss in olfactory epithelium and lung injury by enhancing autophagy in rats with allergic rhinitis
}

\author{
Jinyu Mei ${ }^{1 \S, *}$, Hua Kong ${ }^{2,}$, Zhentao Zhao ${ }^{1}$, Ziyu Chen ${ }^{3}$, Yatang Wang ${ }^{1}$, Jianming Yang ${ }^{1}$ \\ ${ }^{1}$ Department of Otorhinolaryngology Head and Neck Surgery, the Second Affiliated Hospital of Anhui Medical University, Hefei, \\ Anhui, China: \\ ${ }^{2}$ Department of Pharmacology, School of Basic Medical Sciences, Anhui Medical University, Hefei, Anhui, China; \\ ${ }^{3}$ The Second Clinical College of Medicine, Anhui Medical University, Hefei, Anhui, China.
}

\begin{abstract}
Summary Shufengjiedu capsules (SFJDCs), a traditional Chinese medicine, have been widely used as an antiviral, antibacterial, antitumor, and anti-inflammatory drug. However, the roles of SFJDCs in allergic rhinitis remain unclear. The purpose of this study was to investigate the effects of SFJDCs in olfaction and lung injury in rats with allergic rhinitis. An animal model of allergic rhinitis was created by intraperitoneal injection and intranasal administration of ovalbumin to rats. All rats were divided into seven groups: a model group, a low-dose SFJDC group, a medium-dose SFJDC group, a high-dose SFJDC group, a cetirizine group, and a control group. Hematoxylin and eosin (HE) staining was used to observe pathological changes in rat lung and olfactory epithelium (OE) tissue, and peripheral blood was collected and subjected to an enzyme-linked immunosorbent assay (ELISA) to detect IgE, tumor necrosis factor alpha (TNF- $\alpha$ ), and IL-1 $\beta$ levels. Western blotting, immunohistochemistry staining, and immunofluorescence staining were performed to detect inflammatory cytokines and levels of the autophagy biomarker beclin 1 and the apoptosis biomarker cleaeved-caspased 3 in lung and OE tissue. ELISA indicated that SFJDCs significantly decreased IgE, TNF- $\alpha$, and IL-1 $\beta$ levels in peripheral blood, the lungs, and OE tissue. In addition, Western blotting and staining indicated that SFJDCs repair lung injury, protect against neuronal apoptosis in $\mathrm{OE}$, and rescue impaired autophagy in the lungs and $\mathrm{OE}$ tissue. In conclusion, results indicated that SFJDCs might protect against neuronal loss in the $\mathrm{OE}$ and lung injury by enhancing autophagy and decreasing apoptosis in rats with allergic rhinitis. Therefore, SFJDCs might serve as an alternative treatment for allergic rhinitis.
\end{abstract}

Keywords: Shufengjiedu capsules, allergic rhinitis, inflammation, autophagy, apoptosis

\section{Introduction}

Allergic rhinitis (AR) is the most common otorhinolaryngological disease (1). It is also one of

Released online in J-STAGE as advance publication December 21, 2019.

${ }^{\S}$ These authors contributed equally to this work.

*Address correspondence to:

Dr. Jinyu Mei, Department of Otorhinolaryngology Head and Neck Surgery, the Second Affiliated Hospital of Anhui Medical University, 678 Furong Road, Hefei, Anhui 230601, China.

E-mail:meijinyu@ahmu.edu.cn the main causes of olfactory dysfunction (2). The primary clinical manifestations of AR include nasal blockage, a runny and itching nose, sneezing, and olfactory dysfunction (1). AR is an IgE-mediated type I hypersensitivity inflammatory disease of the nasal mucosa (3). IgE bound to FcRI on mast cells and eosinophils is cross-linked by allergens, resulting in the release of diverse preformed and newly synthesized mediators to promote the local recruitment and activation of leukocytes and the production of inflammatory cytokines and T helper 2 (T2) cytokines, which contribute to the development of late-phase reactions (3). Current treatments for AR include 
antihistamines and hormones, but these drugs often have many untoward effects.

In China, traditional Chinese medicine (TCM) formulations, such as Radix isatidis and Radix bupleuri, have been extensively used to treat various respiratory infectious diseases. Shufengjiedu capsules (SFJDCs) are a TCM formulation consisting of eight medicinal herbs, Polygonum cuspidatum Sieb. et Zucc., Forsythia suspense (Thunb) Vahl, Isatis indigotica Fort., Bupleurum chinense DC., Thlaspi arvense L., Verbena officindlis (Officinalis) L., Phragmites communistrin Trin, and Glycyrrhiza uralensis Fisch., that are clinically effective at treating upper respiratory tract infections and ALI/ARDS (4). Since SFJDCs have antiviral action, the China Food and Drug Administration (CFDA) has recommended SFJDCs as a treatment for H1N1- and H5N9-induced acute lung injury (5). In China, SFJDCs have been listed as a drug to combat avian influenza (4,6-8). Studies have reported that active constituents of SFJDCs such as resveratrol and quercetin may alleviate inflammation by suppressing the expression of inflammatory factors via the mitogen-activated protein kinase (MAPK)/nuclear factor- $\kappa \mathrm{B}(\mathrm{NF}-\kappa \mathrm{B})$ signaling pathway (9-11).

Autophagy is an intracellular self-degradative process that is responsible for the systematic degradation and recycling of cellular components such as misfolded or accumulated proteins and damaged organelles (12). One of the pivotal contributions of autophagy to immunity is the cell autonomous control of inflammation. This property leads to systemic consequences and thereby influences the development of innate and adaptive immunity, which promotes or suppresses pathology in various disease contexts. Autophagy can prevent excessive inflammasome activation and reduce the release of inflammatory factors such as TNF- $\alpha$ and IL-1 $\beta$ (13).

Olfactory dysfunction is one of the most common symptoms of AR (14-16). Proinflammatory cytokines (IL-1 $\beta$, TNF- $\alpha$, IL-18, IL-22, and IL-33) and OVAspecific IgE levels in peripheral blood are reported to be significantly increased in guinea pigs with ovalbumin (OVA)-induced AR (17). OVA can induce lung injury, which is characterized by acute hypoxemic respiratory failure with several potential causes that include trauma, shock, viruses, and bacterial endotoxins (18). Cells are increasingly exposed to intracellular oxidative stress and the downstream inflammatory pathway is triggered, leading to lung injury $(19,20)$. When clinically used to treat influenza, SFJDCs can effectively improve symptoms such as nasal congestion, a runny nose, and headaches. Bupleurum, licorice, and other traditional Chinese medicines are important components of SFJDCs (4) and could be used to treat AR. However, few studies have examined the effect of SFJDCs on AR, especially in olfaction. The mechanism of SFJDCs on olfaction and lung injury remain unclear. Thus, the current study sought to explore the effects of treatment with SFJDCs in olfactory epithelium (OE) and the lungs of rats with AR.

The aim of the current study was determine if SFJDCs significantly decrease TNF- $\alpha$ and IL- $1 \beta$ levels and apoptosis, rescue impaired autophagy, and protect against neuronal loss in OE. If SFJDCs repair lung injury and improve olfactory function by enhancing autophagy in rats with AR, then SFJDCs might serve as an alternative treatment for AR.

\section{Materials and Methods}

\subsection{Animals and Materials}

SFJDCs were donated by Jiren Pharmaceutical (Anhui, China). Sixty specific-pathogen-free male SpragueDawley rats weighing $250 \pm 20 \mathrm{~g}$ were purchased from Anhui Medical University's Animal Research Center (Anhui, China). These animals were fed in cages with a 12-hour light/dark cycle, constant temperature, and constant humidity in which mice were given food and water ad libitum. All animals involved in experiments were sacrificed in accordance with international standards. Animal care procedures were approved by the Institutional Animal Care and Use Committee (IACUC) of Anhui Medical University in accordance with the guidelines of the National Institutes of Health (Approval number: LLSC20190513).

\subsection{An animal model of OVA-induced AR and drug administration}

A previously reported protocol was followed to create an animal model of AR (21). The experiment timeline is shown in Figure 1A. The rats were randomly divided into six groups (6 mice per group per cage): a control group (Control), a model group (Model), a cetirizine (Yiling Pharmaceutical Company, Shijiazhuang, China) group (Lev, $0.75 \mathrm{~g} / \mathrm{kg}$ ), a high-dose SFJDC (Jiren pharmaceutical Company, Anhui, China) group (SFJDC-H, $0.18 \mathrm{~g} / \mathrm{kg}$ ), a medium-dose group (SFJDC-M, $0.09 \mathrm{~g} / \mathrm{kg}$ ), and a low-dose group (SFJDC-L, $0.045 \mathrm{~g} /$ $\mathrm{kg}$ ) by gradient dilution. Rats were sensitized (every other day for 14 days) by intraperitoneal injection of 0.3 mg of OVA (Sigma A8040, USA) as an antigen and 30 $\mathrm{mg}$ of aluminum hydroxide as an adjuvant dissolved in $1 \mathrm{~mL}$ of saline. When i.p. immunization concluded, rats were administered $0.25 \%$ OVA dissolved in saline via aerosol (days 14-18). A nasal antigen challenge (days 21-27) was performed with intranasal dripping of $50 \mu \mathrm{l}$ of 5\% OVA daily for 7 consecutive days. Animals in the control group were administered the same volume of saline. After OVA administration, animals in the cetirizine group and SFJDC groups were administered cetirizine or different doses of SFJDCs (twice/day for 14 consecutive days). The dosages of cetirizine and SFJDCs are the clinical recommended doses according to the 
Chinese Pharmacopoeia. Moreover, combined symptom and behavior scoring was used to evaluate the model (21). Serum levels of cytokines were measured using ELISA performed with commercially available kits. Blood samples and nasal mucosa were collected for various assays.

\subsection{Hematoxylin and eosin $(H \& E)$ Staining}

Turbinate bone and lungs embedded in paraffin were sliced at a thickness of $4 \mu \mathrm{m}$. Paraffin sections were deparaffinized in xylene, rehydrated in serial dilutions of alcohol, and immersed in distilled water for $30 \mathrm{sec}$. Sections were dipped in a hematoxylin solution and agitated for $10 \mathrm{~min}$ and then rinsed with distilled water for $1 \mathrm{~min}$. Sections were subsequently stained with a $1 \%$ eosin solution for $10 \mathrm{sec}$ with agitation. Stained sections were dehydrated with increasing concentrations of alcohol (70\% and $90 \%$ alcohol) for $10 \mathrm{~min}$ at a time and then immersed in eosin for $3 \mathrm{~min}$. The sections were dehydrated with alcohol and immersed in xylene. Stained sections were observed using an electron microscope (Olympus, Japan).

\subsection{Toluidine blue staining}

Turbinate bone embedded in paraffin was sliced at a thickness of $4 \mu \mathrm{m}$. Paraffin sections were de-paraffinized in xylene, rehydrated in serial dilutions of alcohol, and immersed in a toluidine blue solution for $30 \mathrm{~min}$. Then sections were dipped in a glacial acetic acid solution and rinsed with distilled water for $1 \mathrm{~min}$. The sections were dehydrated with alcohol and immersed in xylene. Stained sections were observed using an electron microscope (Olympus, Japan) (22).

\subsection{Immunohistochemistry}

For olfactory epithelium staining, animals were first sacrificed and decapitated. Skin and brain tissue were removed, and then the turbinate was removed and immersed in a $0.5 \mathrm{M}$ EDTA $(\mathrm{pH} 8.0)$ solution at room temperature (RT) for 2 weeks. Paraffin-embedded OE tissue was sectioned into $4-\mu \mathrm{m}$-thick slices. All tissue sections were mounted on glass slides. The slides were incubated for 12 hours at $4^{\circ} \mathrm{C}$ temperature with TNF- $\alpha$ (Abcam, ab62609) primary antibody in a humidified chamber, washed, and incubated for 50 min with goat anti-rabbit (074-1505, KPL) secondary antibody. Sections were analyzed with a microscope using a 20 and 40-fold magnification. Cell counting was performed with Image $\mathrm{J}$ whereby the cell number was normalized to the neuronal layer area (23).

\subsection{Immunofluorescent staining}

The OE and lung sections were incubated with beclin-1
(Affinity, AF5128), cleaved-caspase3 (Affinity, AF7022) and IL-1 $\beta$ (Affinity, AF5103) primary antibodies overnight at $4^{\circ} \mathrm{C}$, washed with PBS, and then incubated in second antibodies at $37^{\circ} \mathrm{C}$ for $50 \mathrm{~min}$. After the sections were washing with PBS, they were incubated in DAPI Staining Solution (Beyotime, C1005) for $10 \mathrm{~min}$. The sections were washed with PBS. Afterwards, the slides were mounted with Antifade Mounting Medium (Beyotime, P0126-5ml), viewed, and photographed under a fluorescence microscope (Leica, Germany), and the images were evaluated using the software ImageJ (NIH, Bethesda, MD, USA).

\subsection{Western blotting}

Rats were dissected on ice, and tissue was homogenized in lysis buffer containing $50 \mathrm{mM}$ Tris, $\mathrm{pH} 7.5,150$ $\mathrm{mM} \mathrm{NaCl}, 1 \%$ SDS P40, 5 mM EDTA, and protease inhibitors (Complete Mini; Roche). Cellular debris was removed by centrifugation at $14,000 \mathrm{rpm}$ for 20 min at $4^{\circ} \mathrm{C}$, and the supernatant was collected for analysis. Sodium dodecylsulphate polyacrylamide gel electrophoresis (SDS-PAGE) was performed using a 5\% stacking gel and a $10 \%$ separation gel to separate $20 \mathrm{~L}$ of protein in running buffer. The stacking gel was run at 80 $\mathrm{V}$ for $0.5 \mathrm{~h}$, and the separation gel was run at $100 \mathrm{~V}$ for $1.5 \mathrm{~h}$. The separated proteins were then electrotransferred onto a nitrocellulose filter membrane in transfer buffer at $180 \mathrm{~mA}$ for $1.5 \mathrm{~h}$. Protein blots were blocked with $5 \%$ defatted milk for $30 \mathrm{~min}$ and probed with specific antibodies against TNF- $\alpha$ (Abcam, ab62609), IL-1 $\beta$ (Affinity, AF5103), Beclin-1 (Affinity, AF5128), and cleaved-caspase 3 (Affinity, AF7022). Anti- $\alpha$-tubulin antibody (Sigma, T6557) was used as a loading control. After three washes with TBST, secondary antibody (CWS) was added at room temperature for $1 \mathrm{~h}$ using $5 \%$ milk in TBST followed by three additional washes with TBST. Bands were visualized using the Immobilon Western ECL system and were analyzed with the software Gel Pro Analysis (24).

\subsection{Statistical analysis}

Data were analyzed using one-way ANOVA or a two-sample $t$-test, where $p<0.05$ was considered statistically significant. All data are expressed as the mean \pm SEM.

\section{Results}

\subsection{SFJDCs decreased increased serum IgE and mast cells in OE tissue}

Nasal rubbing and watery rhinorrhea are two common symptoms of AR in rats (1). In order to detect the effects of SFJDCs on these symptoms, an experiment was performed along the timeline shown in Figure 
1A. The frequency of sneezing and nasal rubbing was observed and recorded. All animals were closely observed for development of any nasal symptoms such as sneezing or watery rhinorrhea. In comparison to the control group, mice in the AR model group had clear nasal secretions and an increased frequency of nasal rubbing (data not shown). Figure 1B shows that rats with OVA-induced AR had more watery rhinorrhea. As shown in Figure 1C, IgE levels were lower in the SFJDC-M group than those in the model group but did not differ significantly from those in the SFJDC-L and SFJDC-H groups. As shown in Figure 1D, treatment with SFJDCs significantly reduced the number of mast cells in OE tissue. These findings indicate that SFJDCs alleviated allergic symptoms in rats with AR.

\subsection{Morphological changes in lungs and $O E$ tissue}

As shown in Figure 2A, the model group displayed capillary congestion, obstruction of small airways by lymphocytic infiltrates, and widened alveolar septa in comparison to the control group. Treatment with medium-dose SFJDCs $(0.09 \mathrm{~g} / \mathrm{kg})$ significantly reduced histological injury, such as decreasing obstruction of

A

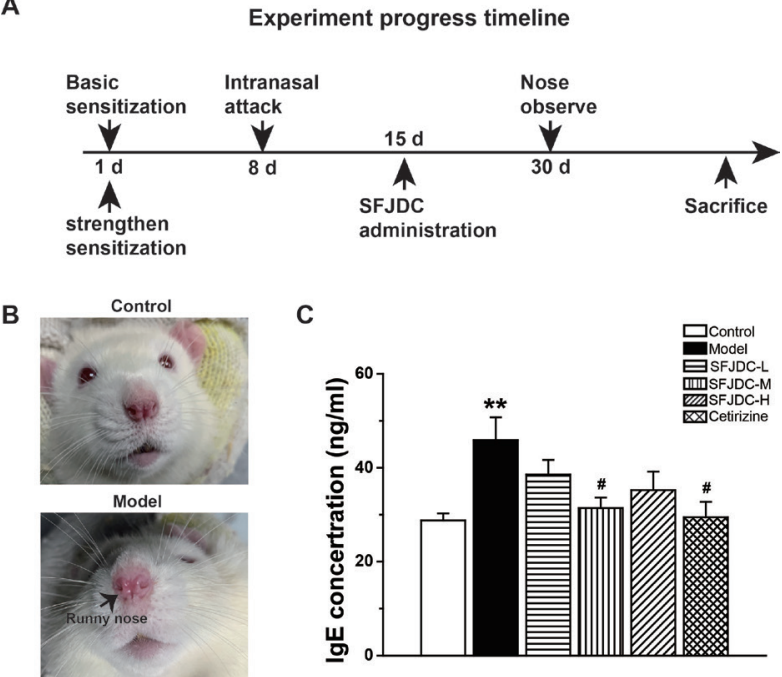

D

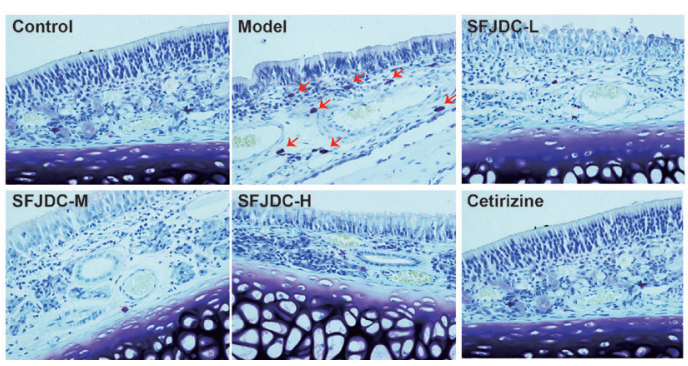

Figure 1. SFJDCs reduced levels of IgE and the number of mast cells in rats with AR. (A): Schematic overview of the study design. (B): Watery rhinorrhea in the model group. (C): Bar graph of the IgE level in serum according to ELISA. (D): Toluidine blue staining of OE from different groups. Arrows indicate mast cells. Data are representative of at least three separate experiments. (**p $<0.01 v s$. control group; ${ }^{*} p<0.05$ vs. model group). small airways, to different degrees. As shown in Figure 2B and $\mathrm{C}, \mathrm{OE}$ appeared thinner and had fewer neurons than that in the control group. The number of neurons in OE increased in the SFJDC-M and cetirizine groups.

\subsection{Levels of TNF- $\alpha$ and $I L-1 \beta$ in serum, the lungs, and OE tissues}

As shown in Figure 3A, ELISA was used to determine levels of TNF- $\alpha$ and IL-1 $\beta$ in serum in each group. Results indicated that TNF- $\alpha$ and IL- $1 \beta$ levels increased significantly in the model group compared to those in the control group. Rats treated with SFJDCs had a marked decrease in levels of TNF- $\alpha$ and IL-1 $\beta$. As shown in Figure 3B, Western blotting was used to detect levels of TNF- $\alpha$ and IL- $1 \beta$ expression in lung tissue. Results indicated that levels of TNF- $\alpha$ and IL-1 $\beta$ protein increased in the model group in comparison to those in the control group. SFJDCs caused a marked decrease in the levels of TNF- $\alpha$ and IL- $1 \beta$ expression in each group. As shown in Figure 3C, immunohistochemistry staining was used to quantify the TNF- $\alpha$ expression in OE tissue in different groups. Results indicated that TNF- $\alpha$ levels decreased significantly in the SFJDC-H and

A

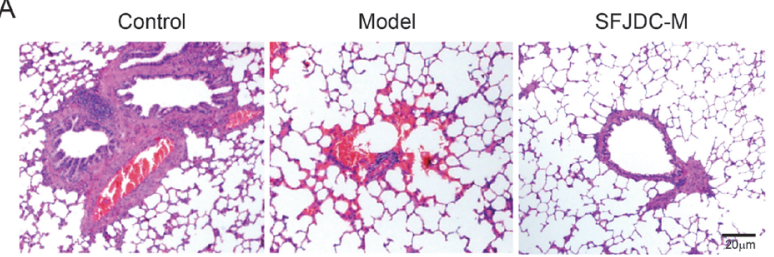

B
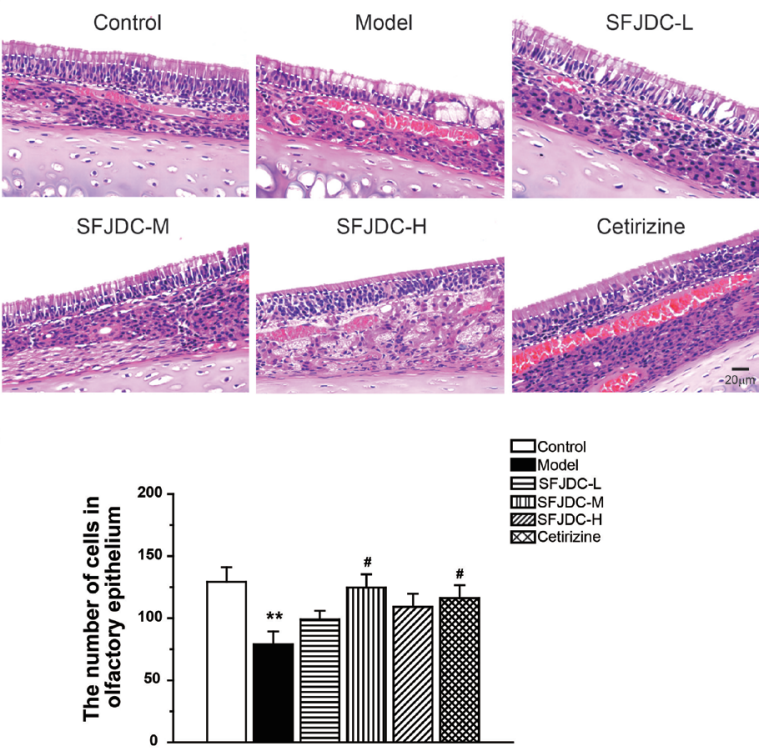

Figure 2. SFJDCs protect against lung injury and $\mathrm{OE}$ neuronal loss in rats with AR. (A): HE staining of lung tissue from the control, model, and SFJDC-M groups. (B): HE staining of OE from the control, model, SFJDC-L, SFJDC-M, SFJDC-H, and cetirizine groups. (C): Bar graph of cell numbers in OE. Data are representative of at least three separate experiments. $\left(* * p<0.01\right.$ vs. control group; ${ }^{*} p<0.05$ vs. model group). 

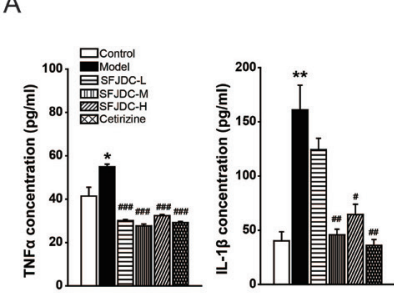

C

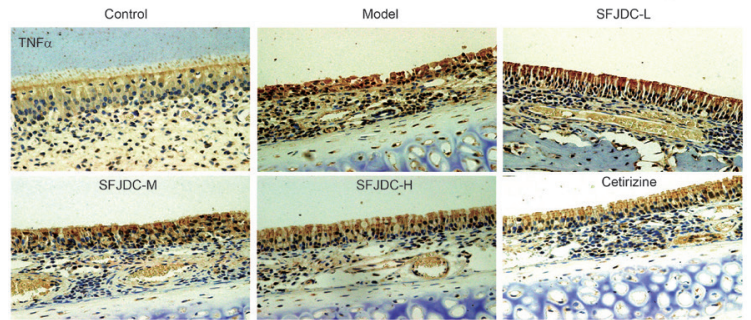

D

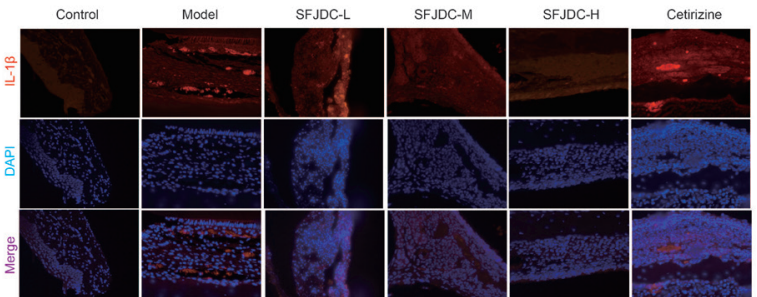

Figure 3. SFJDCs reduced increased TNF- $\alpha$ and IL-1 $\beta$ levels in serum, the lungs, and OE. (A): Bar graph of TNF- $\alpha$ and IL-1 $\beta$ concentrations in serum detecting with ELISA. (B): Western blot bands of TNF- $\alpha$ and IL-1 $\beta$ protein in lung tissue. Bar graph of levels of TNF- $\alpha$ and IL-1 $\beta$ protein in lung tissue. (C): Immunohistochemistry staining of TNF- $\alpha$ in $\mathrm{OE}$ tissue. (D): Immunofluorescent staining of IL-1 $\beta$ in OE tissue. Data are representative of at least three separate experiments. $(* p<$ $0.05,{ }^{* *} p<0.01 v s$. control group; ${ }^{\#} p<0.05,{ }^{\#} p<0.01,{ }^{*} p<$ 0.001 vs. model group).

cetirizine groups. There were no significant differences in those levels in the SFJDC-L and SFJDC-M groups. As shown in Figure 3D, immunofluorescent staining was performed to detect the level of IL- $1 \beta$ in OE tissue. Red fluorescence for IL-1 $\beta$ increased in the model group in comparison to that the control group, and red fluorescence decreased in the SFJDC-M and SFJDC-H groups. The results shown in Figure 3 indicate that SFJDCs decreased inflammation levels in rats with AR.

\subsection{Levels of autophagy and apoptosis in the lungs and OE tissue}

As shown in Figure 4, red fluorescence for beclin1 was not evident in the model group, indicating a very low level of autophagy. Green fluorescence for caspase 3 increased in the model group in comparison to that in the control group, indicating that the level of apoptosis increased in the model group. When rats were treated with SFJDCs, the beclin1 level significantly increased in the SFJDC-M and SFJDC-H groups. A lower level of caspase3 was observed only in the SFJDC-L group. When Western blotting was used to detect levels of beclin 1 and caspase 3 protein expression, the same trend was observed, indicating that SFJDCs increased the level of autophagy and decreased apoptosis. Since OE lesions also appeared, as shown in Figure 5, immunofluorescent staining was used to detect beclin1 and caspase 3 levels in OE tissue. Results indicated that the beclin1 level decreased and the caspase 3 level increased in the model group in comparison to levels in the control group. SFJDCs resulted in a marked increase in the level of beclin 1 and a marked decrease in the level of caspase 3 in the SFJDC-M and SFLDC-H groups.

\section{Discussion}

The current study found that SFJDCs reduce neuronal loss in $\mathrm{OE}$ and protect against lung injury induced by AR by increasing the level of autophagy and reducing apoptosis and inflammation factors in rats with AR. These findings are corroborated by several lines of evidence: (i) SFJDCs ameliorate the symptoms of AR, such as a runny or itching nose, sneezing, a decrease in the number of mast cells, and a lower level of IgE (which are markers of an allergy); (ii) TNF $\alpha$ and IL-1 $\beta$ levels in serum, OE, and lung tissues are downregulated by treatment with SFJDCs; (iii) SFJDCs rescue the levels of beclin 1 and cleaved-caspase 3 protein, which are respective biomarkers of autophagy and apoptosis. Together, these findings indicate that SFJDCs may play a critical role in AR.

In order to study the effects of SFJDCs on AR, the first step in the current study was to create a reliable animal model of AR. OVA is mainly used as an allergen to create animal models of AR (25-27). The primary clinical manifestations of AR include nasal blockage, a runny and itching nose, sneezing, and olfactory dysfunction (1). In the current study, rats had a runny and itching nose and sneezing after OVA treatment (Figure 1B). A high level of IgE and an increase in mast cells are common hallmarks of AR. Mast cells release molecules that can lead to an allergic reaction. Treatment with SFJDCs decreased nasal rubbing and watery rhinorrhea in rats with AR. Together, these findings indicate that an animal model of AR was successfully created and that SFJDCs ameliorated some symptoms of AR.

OVA is mainly used as an allergen to create animal models of AR (21). In the current study, however, OVAinduced AR produced pathological changes in rats not only in olfactory tissue but also in the lungs (Figure 2AC). Although several studies have found that asthma and rhinitis are characterized by a similar inflammatory process (28-30), pathophysiologic interactions between the upper and lower airways are not entirely understood. The condition of the upper airway definitely influences the lower airway. The current study considered whether lung injury was caused by AR. AR triggers a systemic increase in inflammation (31). Numerous studies have indicated that the active constituents of SFJDCs, such as resveratrol and other flavonoids, inhibit acute lung injury (ALI). In addition, the MAPK and NF-kB signaling 


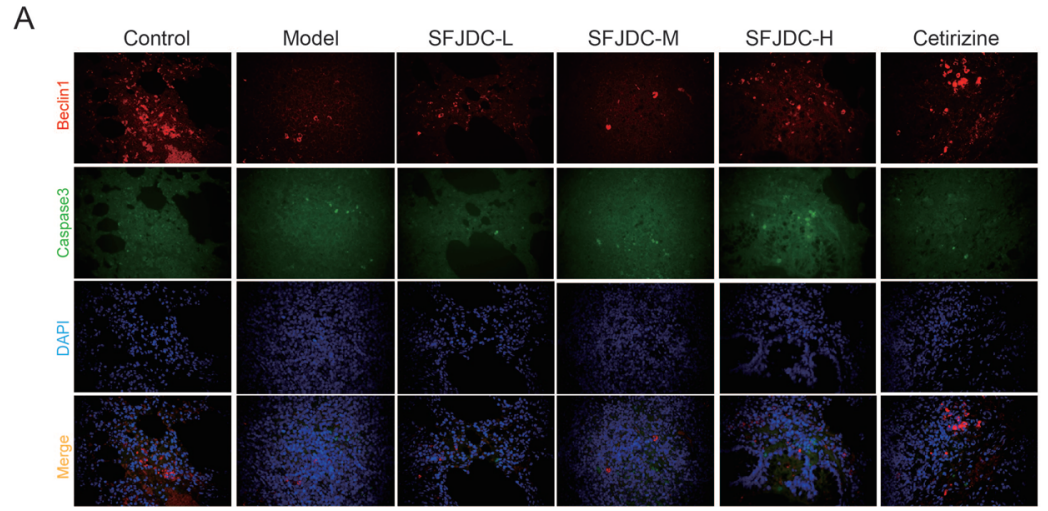

B
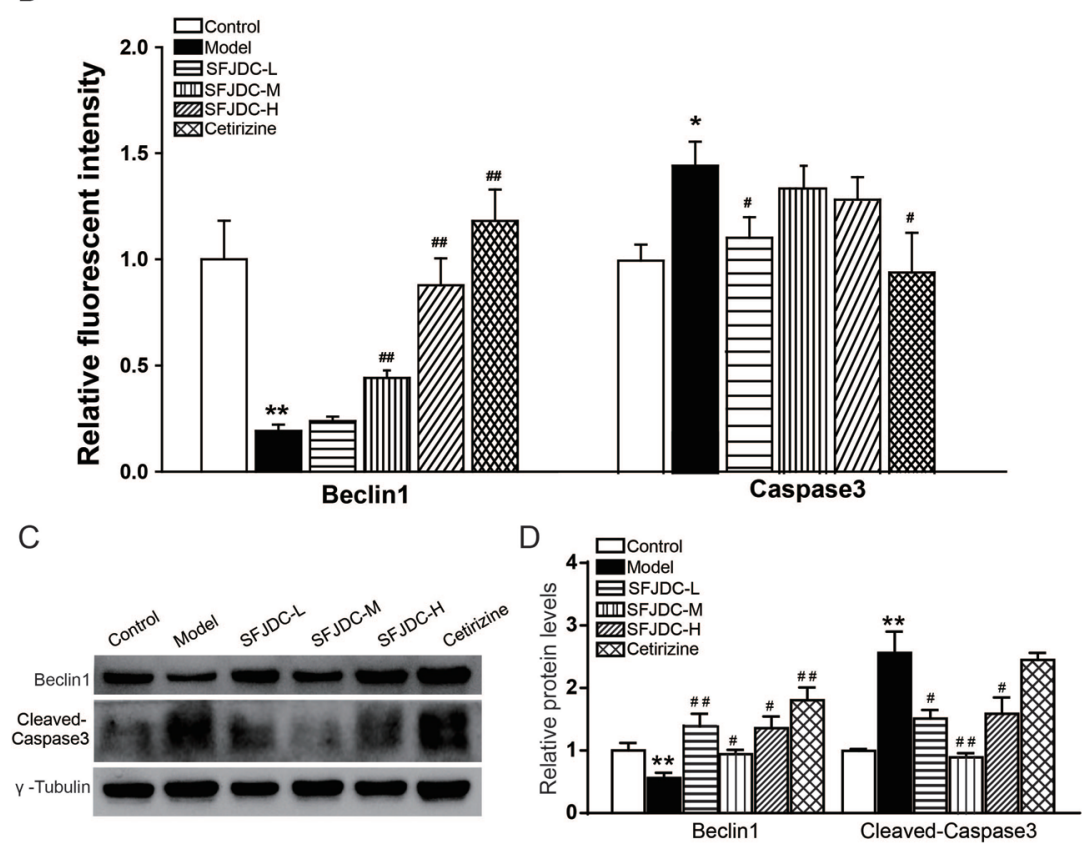

Figure 4. SFJDCs rescued impaired autophagy and levels of apoptosis in lung tissue. (A): Immunofluorescent staining of beclin1 and cleaved-caspase 3 in lung tissue. (B): Bar graph of levels of beclin1 and cleaved-caspase 3 expression in lung tissue. (C): Western blot bands of beclin1 and cleaved-caspase3 protein in lung tissue. (D): Bar graph of Western blot bands in Figure 5 (C). Data are representative of at least three separate experiments. $\left({ }^{*} p<0.05,{ }^{* *} p<0.01\right.$ vs. control group; ${ }^{\#} p<0.05,{ }^{\# \#} p<0.01$ vs. model group).

pathways may be involved in ALI (32-38). NF- $\mathrm{kB}$ is a key factor in the inflammatory response and is involved in the release of inflammatory factors and development of ALI $(39,40)$. IL-1 $\beta$ and TNF- $\alpha$ may serve as biomarkers of the NF- $\kappa$ B inflammatory pathway. SFJDCs are reported to alleviate inflammation by inhibiting IL-1 $\beta$ in the NF-kB signaling pathway. The current study found that IL- $1 \beta$ and TNF- $\alpha$ levels decreased significantly when rats were treated with SFJDCs, indicating that SFJDCs ameliorate AR by regulating inflammation levels. Autophagy can prevent excessive inflammasome activation and reduce the release of inflammatory factors such as TNF- $\alpha$ and IL-1 $\beta$ (13). In a physiological state, autophagy can promote apoptosis. Examination of nasal lavage fluid from rats with AR has revealed apoptosis of eosinophils. However, the role of autophagy in AR still remain unknown. Measurement of beclin1 and caspase 3 protein levels revealed that SFJDCs improved levels of autophagy and inhibited apoptosis in OE and lung tissue. These findings indicate that SFJDCs may alleviate lung injury by mediating autophagy and apoptosis and by reducing the release of inflammatory factors in lung tissue.

Olfactory function is reported to be impaired in rats with AR (14). The current study used HE staining to observe the pathology of OE tissue in rats. Rats treated with SFJDCs had more neurons in OE tissue. The immunofluorescent staining of caspase 3 indicated that SFJDCs markedly decreased apoptosis in OE tissue. Inflammatory factors such as TNF- $\alpha$ and IL-1 $\beta$ can induce an increase in apoptosis. Like rats treated with cetirizine, rats treated with SFJDCs had a marked decrease in inflammatory factors and levels of apoptosis in OE tissue. The level of autophagy increased when rats were treated with SFJDCs. These findings indicate that SFJDCs may protect against neuronal loss in OE 
A
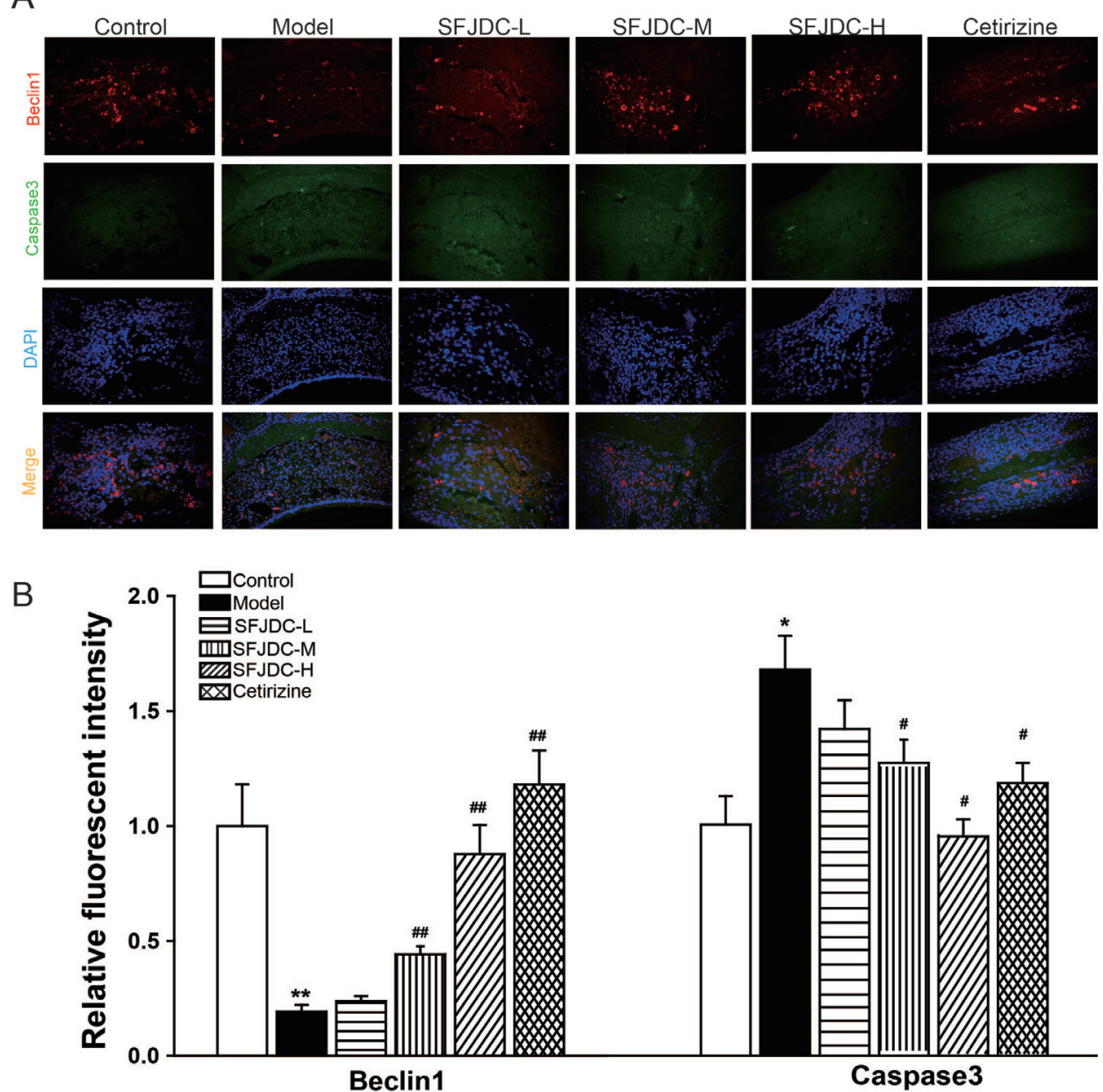

Figure 5. SFJDCs rescued impaired autophagy and levels of apoptosis in OE tissue. (A): Immunofluorescent staining of beclin1 and cleaved-caspase 3 in OE tissue. (B): Bar graph of levels of beclin1 and cleaved-caspase 3 expression in OE tissue. Data are representative of at least three separate experiments. ${ }^{*} p<0.05,{ }^{*} p<0.01 v s$. control group; ${ }^{*} p<0.05,{ }^{\#} p<0.01 v s$. model group).

by mediating autophagy, apoptosis, and the release of inflammatory factors.

In conclusion, this study revealed that SFJDCs alleviate OVA-induced AR in a rat model by mediating levels of autophagy and apoptosis and by suppressing the release of inflammatory factors. Thus, SFJDCs might serve as an alternative medication to inhibit inflammation in OE and lung tissue, and SFJDCs have great potential for widespread clinical use.

\section{Acknowledgements}

This work was financially supported by grants from the Major Basic Research Program in Universities of Anhui (No. KJ2018ZD021). The authors wish to thank Wanpeng Lu and Xiangming Tao for providing technical support.

\section{Statement of Ethics}

Animal care procedures were approved by the Institutional Animal Care and Use Committee (IACUC) at Anhui Medical University in accordance with the guidelines of the National Institutes of Health.

\section{References}

1. Bousquet J, Khaltaev N, Cruz AA, e al. Allergic Rhinitis and its Impact on Asthma (ARIA) 2008 update (in collaboration with the World Health Organization, GA(2) LEN and AllerGen). Allergy. 2008; 63 Suppl 86:8-160.

2. Guilemany JM, García-Piñero A, Alobid I, Cardelús S, Centellas S, Bartra J, Valero A, Picado C, Mullol J. Persistent allergic rhinitis has a moderate impact on the sense of smell, depending on both nasal congestion and inflammation, Laryngoscope. 2009; 119:233-238

3. Wei-Xu H, Wen-Yun Z, Xi-Ling Z, Zhu W, Li-Hua W, Xiao-Mu W, Hui-Ping W, Wen-Ding W, Dan H, Qin $\mathrm{X}$, Guo-Zhu H. Anti-Interleukin-1 beta/tumor necrosis ractor-alpha $\operatorname{IgY}$ antibodies reduce pathological allergic responses in Guinea pigs with allergic rhinitis. Mediators Inflamm. 2016; 2016:3128182.

4. Tao Z, Gao J, Zhang G, Xue M, Yang W, Tong C, Yuan Y. Shufeng Jiedu Capsule protect against acute lung injury by suppressing the MAPK/NF-kappaB pathway. Biosci Trends. 2014; 8:45-51. 
5. Tao Z, Yang Y, Shi W, Xue M, Yang W, Song Z, Yao C, Yin J, Shi D, Zhang Y, Cai Y, Tong C, Yuan Y. Complementary and alternative medicine is expected to make greater contribution in controlling the prevalence of influenza. Biosci Trends. 2013;7:253-256.

6. Bao Y, Gao Y, Cui X. Effect of Shufeng Jiedu capsules as a broad-spectrum antibacterial. Biosci Trends. 2016; 10:74-78.

7. Cheng L, Li F, Ma R, Hu X. Forsythiaside inhibits cigarette smoke-induced lung inflammation by activation of Nrf2 and inhibition of NF- $\mathrm{KB}$. Int Immunopharmacol. 2015; 28:494-499.

8. Li Y, Chang N, Han Y, Zhou M, Gao J, Hou Y, Jiang M, Zhang T, Bai G. Anti-inflammatory effects of Shufengjiedu capsule for upper respiratory infection via the ERK pathway. Biomed Pharmacother. 2017; 94:758766.

9. Busch F1, Mobasheri A, Shayan P, Lueders C, Stahlmann $\mathrm{R}$, Shakibaei M. Resveratrol modulates interleukin-1 $\beta$ induced phosphatidylinositol 3-kinase and nuclear factor $\kappa \mathrm{B}$ signaling pathways in human tenocytes. J Biol Chem. 2012; 287:38050-380563.

10. Gao J, Inagaki Y, Li X, Kokudo N, Tang W. Research progress on natural products from traditional Chinese medicine in treatment of Alzheimer's disease. Drug Discov Ther. 2013; 7:46-57.

11. Song Y, Liu J, Zhang F, Zhang J, Shi T, Zeng Z. Antioxidant effect of quercetin against acute spinal cord injury in rats and its correlation with the p38MAPK/ iNOS signaling pathway. Life Sci. 2013; 92:1215-1221.

12. Glick D, Barth S, Macleod KF. Autophagy: Cellular and molecular mechanisms. J Pathol. 2010; 221:3-12.

13. Deretic V, Levine B. Autophagy balances inflammation in innate immunity. Autophagy. 2018; 14:243-251.

14. Ozaki S, Toida K, Suzuki M, Nakamura Y, Ohno N, Ohashi T, Nakayama M, Hamajima Y, Inagaki A, Kitaoka $\mathrm{K}$, Sei H, Murakami S. Impaired olfactory function in mice with allergic rhinitis. Auris Nasus Larynx. 2010; 37:575-583.

15. Mott AE, Cain WS, Lafreniere D, Leonard G, Gent JF, Frank ME. Topical corticosteroid treatment of anosmia associated with nasal and sinus disease. Arch Otolaryngol Head Neck Surg. 1997; 123: 367-372.

16. Simola M, Malmberg H. Sense of smell in allergic and nonallergic rhinitis. Allergy. 1998; 53:190-194.

17. Guo-Zhu H, Xi-Ling Z, Zhu W, Li-Hua W, Dan H, Xiao$\mathrm{Mu} \mathrm{W}$, Wen-Yun Z, Wei-Xu H. Therapeutic potential of combined anti-IL-1beta IgY and anti-TNF-alpha IgY in guinea pigs with allergic rhinitis induced by ovalbumin. Int Immunopharmacol. 2015; 25:155-161.

18. Vlaar AP, Juffermans NP. Transfusion-related acute lung injury: A clinical review. Lancet. 2013; 382:984-994.

19. Ellison MA, Ambruso DR, Silliman CC. Therapeutic options for transfusion related acute lung injury: The potential of the G2A receptor. Curr Pharm Des. 2012; 18:3255-3259.

20. Ward PA. Oxidative stress: Acute and progressive lung injury. Ann N Y Acad Sci. 2010 Aug;1203:53-59.

21. Wang X, Zhu Y, Ni D, Lv W, Gao Z, Qi F. Intranasal application of glucocorticoid alleviates olfactory dysfunction in mice with allergic rhinitis. Exp Ther Med. 2017; 14:3971-3978.

22. Ratna Kumari TVN, Ahmed Mujib BR. Toluidine blue with a synergistic effect in morphological assessment of oral cytosmears. J Cytol. 2018; 35:8-14
23. Zhang J, Hao C, Jiang J, Feng Y, Chen X, Zheng Y, Liu J, Zhang Z, Long C, Yang L. The mechanisms underlying olfactory deficits in apolipoprotein E-deficient mice: Focus on olfactory epithelium and olfactory bulb. Neurobiol Aging. 2018; 62:20-33.

24. Chen M, Wang J, Jiang J, et al. APP modulates KCC2 expression and function in hippocampal GABAergic inhibition. Elife. 2017; 6. pii: e20142.

25. Takahashi N, Aramaki Y, Tsuchiya S. Allergic rhinitis model with Brown Norway rat and evaluation of antiallergic drugs. J Pharmacobiodyn. 1990; 13:414-420.

26. Nakaya M, Dohi M, Okunishi K, Nakagome K, Tanaka R, Imamura M, Baba S, Takeuchi N, Yamamoto K, Kaga $\mathrm{K}$. Noninvasive system for evaluating allergen-induced nasal hypersensitivity in murine allergic rhinitis. Lab Invest. 2006; 86:917-26.

27. Tanaka K, Okamoto Y, Nagaya Y, Nishimura F, Takeoka A, Hanada S, Kohno S, Kawai M. A nasal allergy model developed in the guinea pig by intranasal application of 2,4-toluene diisocyanate. Int Arch Allergy Appl Immunol. 1988; 85:392-397.

28. Bousquet J, Chanez P, Lacoste JY, Barnéon G, Ghavanian N, Enander I, Venge P, Ahlstedt S, Simony-Lafontaine J, Godard P, Michel F. Eosinophilic inflammation in asthma. N Engl J Med. 1990; 323:1033-1039.

29. Bentley AM, Jacobson MR, Cumberworth V, Barkans JR, Moqbel R, Schwartz LB, Irani AM, Kay AB, Durham SR. Immunohistology of the nasal mucosa in seasonal allergic rhinitis: Increases in activated eosinophils and epithelial mast cells. J Allergy Clin Immunol. 1992; 89:877-883

30. Bradley BL, Azzawi M, Jacobson M, Assoufi B, Collins JV, Irani AM, Schwartz LB, Durham SR, Jeffery PK, Kay AB. Eosinophils, T-lymphocytes, mast cells, neutrophils, and macrophages in bronchial biopsy specimens from atopic subjects with asthma: Comparison with biopsy specimens from atopic subjects without asthma and normal control subjects and relationship to bronchial hyperresponsiveness. J Allergy Clin Immunol. 1991; 88:661-674.

31. Borish L. Allergic rhinitis: systemic inflammation and implications for management. J Allergy Clin Immunol. 2003; 112:1021-1031.

32. Meng G, Liu Y, Lou C, Yang H. Emodin suppresses lipopolysaccharide-induced pro-inflammatory responses and NF- $\mathrm{KB}$ activation by disrupting lipid rafts in CD14negative endothelial cells. Br J Pharmacol. 2010; 161:1628-1644

33. Nepal M, Li L, Cho HK, Park JK, Soh Y. Kaempferol induces chondrogenesis in ATDC5 cells through activation of ERK/BMP-2 signaling pathway. Food Chem Toxicol. 2013; 62:238-245.

34. Chien YC, Sheu MJ, Wu CH, Lin WH, Chen YY, Cheng PL, Cheng HC. A Chinese herbal formula "Gan-LuYin" suppresses vascular smooth muscle cell migration by inhibiting matrix metalloproteinase-2/9 through the PI3K/AKT and ERK signaling pathways. BMC Complement Altern Med. 2012; 12:137.

35. Kim A, Yim NH, Im M, Jung YP, Liang C, Cho WK, Ma JY. Ssanghwa-tang, an oriental herbal cocktail, exerts anti-melanogenic activity by suppression of the p38 MAPK and PKA signaling pathways in B16F10 cells. BMC Complement Altern Med. 2013; 13:214.

36. Wu JS, Shi R, Zhong J, Lu X, Ma BL, Wang TM, Zan B, Ma YM, Cheng NN, Qiu FR. Renal Protective 
Role of Xiexin Decoction with Multiple Active Ingredients Involves Inhibition of Inflammation through Downregulation of the Nuclear Factor- $\kappa$ B Pathway in Diabetic Rats. Evid Based Complement Alternat Med. 2013; 2013:715671.

37. Yoo H, Ku SK, Baek YD, Bae JS. Anti-inflammatory effects of rutin on HMGB 1-induced inflammatory responses in vitro and in vivo. Inflamm Res. 2014; 63:197-206.

38. Xia JF, Gao JJ, Inagaki Y, Kokudo N, Nakata M, Tang W. Flavonoids as potential anti-hepatocellular carcinoma agents: Recent approaches using HepG2 cell line. Drug Discov Ther. 2013; 7:1-8.
39. Chi G, Wei M, Xie X, Soromou LW, Liu F, Zhao S. Suppression of MAPK and NF-kB pathways by limonene contributes to attenuation of lipopolysaccharideinduced inflammatory responses in acute lung injury. Inflammation. 2013; 36:501-511.

40. Zhu T, Wang DX, Zhang W, Liao XQ, Guan X, Bo H, Sun JY, Huang NW, He J, Zhang YK, Tong J, Li CY. Andrographolide protects against LPS-induced acute lung injury by inactivation of NF-кB. PLoS One. 2013; 8:e56407

(Received May 17, 2019; Revised September 2, 2019; Rerevised December 4, 2019; Accepted December 15, 2019) 